\title{
ON AN EXTENSION OF A BILATERAL GENERATING FUNCTION INVOLVING GENERALISED BESSEL POLYNOMIALS
}

\author{
P. K. MAITI
}

\begin{abstract}
In this note we obtain an extension of the bilateral generating function involving generalised Bessel polynomials obtained by S. K. Pan from the existence of a quasi-bilinear generating relation involving the polynomial under consideration.
\end{abstract}

\section{Introduction}

In [1], quasi-bilateral generating function is defined as follows:

$$
G(x, u, w)=\sum_{n=0}^{\infty} a_{n} p_{n}^{(\alpha)}(x) q_{m}^{(n)}(u) w^{n}
$$

where $p_{n}^{(\alpha)}(x)$ and $q_{m}^{(n)}(u)$ are two polynomials of orders $n, m$ and of parameters $\alpha, n$ respectively. When $q_{m}^{(n)}(u) \equiv p_{m}^{(n)}(u)$, then the above generating relation is quasi-bilinear.

In [2], Pan obtained the following theorem on bilateral generating functions involving generalised Bessel Polynomials, $Y_{n}^{(\alpha)}(x)$ introduced by H. L. Krall and O. Frink [3]:

Theorem 1. If

$$
G(x, w)=\sum_{n=0}^{\infty} a_{n} Y_{n}^{(\alpha)}(x) w^{n}
$$

then

$$
e^{-\frac{\beta w}{(1-w x)}}(1-w x)^{\alpha-2} G(x(1-w x), w z)=\sum_{n=0}^{\infty} w^{n} f_{n}(x, z)
$$

where

$$
f_{n}(x, z)=\sum_{p=0}^{n} a_{p} \frac{\beta^{n-p}}{(n-p) !} Y_{n}^{(\alpha-2(n-p))}(x) z^{p}
$$

The object of the present paper is to obtain the following theorem as an extension of the above theorem from the concept of quasi-bilinear generating function by using one parameter continuous transformations group.

Received January 15, 2007.

2000 Mathematics Subject Classification. 33A47.

Key words and phrases. Bessel polynomial, quasi-bilateral generating function. 
Theorem 2. If

$$
G(x, u, w)=\sum_{n=0}^{\infty} a_{n} Y_{n}^{(\alpha)}(x) Y_{m}^{(n)}(u) w^{n}
$$

then

$$
\begin{array}{r}
(1-w)^{-m+1}(1-w x)^{\alpha-2} \exp \left(-\frac{\beta w}{1-w x}\right) G\left(x(1-w x), \frac{u}{1-w}, \frac{w v}{1-w}\right) \\
=\sum_{n=0}^{\infty} \sum_{p=0}^{\infty} \sum_{q=0}^{\infty} a_{n} \frac{w^{n+p+q}}{p ! q !} v^{p} \beta^{p} Y_{n+p}^{(\alpha-2 p)}(x)(m+n-1)_{q} Y_{m}^{(n+q)}(u) .
\end{array}
$$

\section{Proof of the Theorem}

In $[4,5]$, we see that

$$
\begin{aligned}
& R_{1}=x^{2} y^{-2} z \frac{\partial}{\partial x}+x y^{-1} z \frac{\partial}{\partial y}+\beta y^{-2} z-2 x y^{-2} z . \\
& R_{2}=u t \frac{\partial}{\partial u}+t^{2} \frac{\partial}{\partial t}+(m-1) t
\end{aligned}
$$

such that

$$
\begin{aligned}
R_{1}\left(Y_{n}^{(\alpha)}(x) y^{\alpha} z^{n}\right) & =\beta Y_{n+1}^{(\alpha-2)}(x) y^{\alpha-2} z^{n+1} \\
R_{2}\left(Y_{m}^{(n)}(u) t^{n}\right) & =(m+n-1) Y_{m}^{(n+1)}(u) t^{n}
\end{aligned}
$$

and

$$
\begin{aligned}
e^{w R_{1}} f(x, y, z)= & \exp \left[-\frac{\beta w y^{-2} z}{1-w x y^{-2} z}\right]\left(1-w x y^{-2} z\right)^{-2} \\
& \times f\left(x\left(1-w x y^{-2} z\right), y\left(1-w x y^{-2} z\right), z\right) \\
e^{w R_{2}} f(u, t)= & (1-w t)^{-m+1} f\left(\frac{u}{1-w t}, \frac{t}{1-w t}\right) .
\end{aligned}
$$

Let we consider

$$
G(x, u, w)=\sum_{n=0}^{\infty} a_{n} Y_{n}^{(\alpha)}(x) Y_{m}^{(n)}(u) w^{n} .
$$

Replacing $w$ by $w z t v$ and then multiplying both sides of (2.5) by $y^{\alpha}$, we get

$$
y^{\alpha} G(x, u, w z t v)=\sum_{n=0}^{\infty} a_{n}(w v)^{n}\left(Y_{n}^{(\alpha)}(x) y^{\alpha} z^{n}\right)\left(Y_{m}^{(n)}(u) t^{n}\right) .
$$

Operating $e^{w R_{1}} e^{w R_{2}}$ on both sides of (2.6), we get

$$
\begin{aligned}
& e^{w R_{1}} e^{w R_{2}}\left(y^{\alpha} G(x, u, w z t v)\right) \\
& \quad=e^{w R_{1}} e^{w R_{2}}\left(\sum_{n=0}^{\infty} a_{n}(w v)^{n}\left(Y_{n}^{(\alpha)}(x) y^{\alpha} z^{n}\right)\left(Y_{m}^{(n)}(u) t^{n}\right)\right) .
\end{aligned}
$$


L.H.S. of (2.7) is

$$
\begin{aligned}
e^{w R_{1}} & e^{w R_{2}}\left(y^{\alpha} G(x, u, w z t v)\right) \\
= & e^{w R_{1}}\left[(1-w t)^{-m+1} y^{\alpha} G\left(x, \frac{u}{1-w t}, \frac{w z t v}{1-w t}\right)\right] \\
= & (1-w t)^{-m+1} \exp \left(-\frac{\beta w y^{-2} z}{1-w x y^{-2} z}\right)\left(1-w x y^{-2} z\right)^{-2} \\
& \quad \times y^{\alpha}\left(1-w x y^{-2} z\right)^{\alpha} G\left(x\left(1-w x y^{-2} z\right), \frac{u}{1-w t}, \frac{w z t v}{1-w t}\right) .
\end{aligned}
$$

R.H.S. of (2.7) is

$$
\begin{aligned}
e^{w R_{1}} & e^{w R_{2}}\left(\sum_{n=0}^{\infty} a_{n}(w v)^{n}\left(Y_{n}^{(\alpha)}(x) y^{\alpha} z^{n}\right)\left(Y_{m}^{(n)}(u) t^{n}\right)\right) \\
& =\sum_{n=0}^{\infty} \sum_{p=0}^{\infty} \sum_{q=0}^{\infty} a_{n} \frac{w^{n+p+q}}{p ! q !} v^{n} R_{1}^{p}\left(Y_{n}^{(\alpha)}(x) y^{\alpha} z^{n}\right) R_{2}^{q}\left(Y_{m}^{(n)}(u) t^{n}\right) \\
& =\sum_{n=0}^{\infty} \sum_{p=0}^{\infty} \sum_{q=0}^{\infty} a_{n} \frac{w^{n+p+q}}{p ! q !} v^{n} \beta^{p} Y_{n+p}^{(\alpha-2 p)}(x) y^{\alpha-2 p} z^{n-2 p}(m+n-1)_{q} Y_{m}^{(n+q)}(u) t^{n+q} .
\end{aligned}
$$

Equation (2.8) and (2.9) and then putting $y=z=t=1$, we get

$$
\begin{aligned}
(1- & w)^{-m+1}(1-w x)^{\alpha-2} \exp \left(-\frac{\beta w}{1-w x}\right) G\left(x(1-w x), \frac{u}{1-w}, \frac{w v}{1-w}\right) \\
& =\sum_{n=0}^{\infty} \sum_{p=0}^{\infty} \sum_{q=0}^{\infty} a_{n} \frac{w^{n+p+q}}{p ! q !} v^{n} \beta^{p} Y_{n+p}^{(\alpha-2 p)}(x)(m+n-1)_{q} Y_{m}^{(n+q)}(u) .
\end{aligned}
$$

which is Theorem 2 .

\section{Particular Case:}

If we put $m=0$ in (2.10), we get

$$
\begin{aligned}
(1- & w)(1-w x)^{\alpha-2} \exp \left(-\frac{\beta w}{1-w x}\right) G\left(x(1-w x), \frac{w v}{1-w}\right) \\
& =\sum_{n=0}^{\infty} \sum_{p=0}^{\infty} \sum_{q=0}^{\infty} a_{n} \frac{w^{n+p+q}}{p ! q !} v^{n} \beta^{p} Y_{n+p}^{(\alpha-2 p)}(x)(n-1)_{q} \\
& =\sum_{n=0}^{\infty} \sum_{p=0}^{\infty} a_{n} \frac{w^{n+p}}{p !} v^{n} \beta^{p} Y_{n+p}^{(\alpha-2 p)}(x) \sum_{q=0}^{\infty} \frac{w^{q}}{q !}(n-1)_{q} \\
& =\sum_{n=0}^{\infty} \sum_{p=0}^{\infty} a_{n} \frac{w^{n+p}}{p !} v^{n} \beta^{p} Y_{n+p}^{(\alpha-2 p)}(x)(1-w)^{-n+1} \\
& =(1-w) \sum_{n=0}^{\infty} \sum_{p=0}^{\infty} a_{n}\left(\frac{v}{1-w}\right)^{n} \frac{w^{n+p}}{p !} \beta^{p} Y_{n+p}^{(\alpha-2 p)}(x) .
\end{aligned}
$$

Therefore,

$$
(1-w x)^{\alpha-2} \exp \left(-\frac{\beta w}{1-w x}\right) G\left(x(1-w x), \frac{w v}{1-w}\right)
$$




$$
=\sum_{n=0}^{\infty} \sum_{p=0}^{\infty} a_{n} w^{n+p} \frac{\beta^{p}}{p !} Y_{n+p}^{(\alpha-2 p)}(x)\left(\frac{v}{1-w}\right)^{n} .
$$

Replacing $\frac{v}{1-w}$ by $v$, we get

$$
\begin{aligned}
(1- & w x)^{\alpha-2} \exp \left(-\frac{\beta w}{1-w x}\right) G(x(1-w x), w v) \\
& =\sum_{n=0}^{\infty} w^{n} \sum_{p=0}^{\infty} a_{n-p} \frac{\beta^{p}}{p !} Y_{n}^{(\alpha-2 p)}(x) v^{n-p} \\
& =\sum_{n=0}^{\infty} w^{n} \sum_{p=0}^{n} a_{p} \frac{\beta^{n-p}}{(n-p) !} Y_{n}^{(\alpha-2(n-p))}(x) v^{p} \\
& =\sum_{n=0}^{\infty} w^{n} f_{n}(x, v),
\end{aligned}
$$

where

$$
f_{n}(x, v)=\sum_{p=0}^{\infty} a_{p} \frac{\beta^{n-p}}{(n-p) !} Y_{n}^{(\alpha-2(n-p))}(x) v^{p}
$$

which is Theorem 1 .

\section{Acknowledgement}

I am grateful to Prof. A. K. Chongchar of BESU, Howrach for his constant encouragement for carrying out research work.

\section{References}

[1] S. K. Chatterjea and S. P. Chakraborty, A unified group-theoretic method of obtaining a more general class of generating relations from a given class of quasi-bilateral (or quasi-bilinear) generating relations involving some special functions, Pure Math. Manuscript 8(1989), 153-162.

[2] S. K. Pan, Application of group-theory in the study of some problems of special functions, Ph.D. Thesis, C.U. (1995).

[3] H. L. Krall and O. Frink, An orthogonal polynomial; The Bessel Polynomials, Trans. Amer. Math. Soc. 65(1949), 100-115.

[4] A. K. Chongdar, On generating functions of Bessel Polynomials by group-theoretic method, Boll. U.M.I. 7(1991), 163-170.

[5] M. P. Chen and C. C. Feng, Group-theoretic origins of certain of certain generating functions of generalised Bessel polynomials, Tamkang Jour. Math. 6(1975), 87-93.

Department of Physics, Bangabasi Evening College, 19, Rajkumar Chakraborty Sarani, Kolkata, Pin - 700 009, W.B., India. 